\title{
Práticas de alimentação complementar no primeiro ano de vida e fatores associados
}

\author{
Complementary feeding practices \\ in the first year of life and \\ associated factors
}

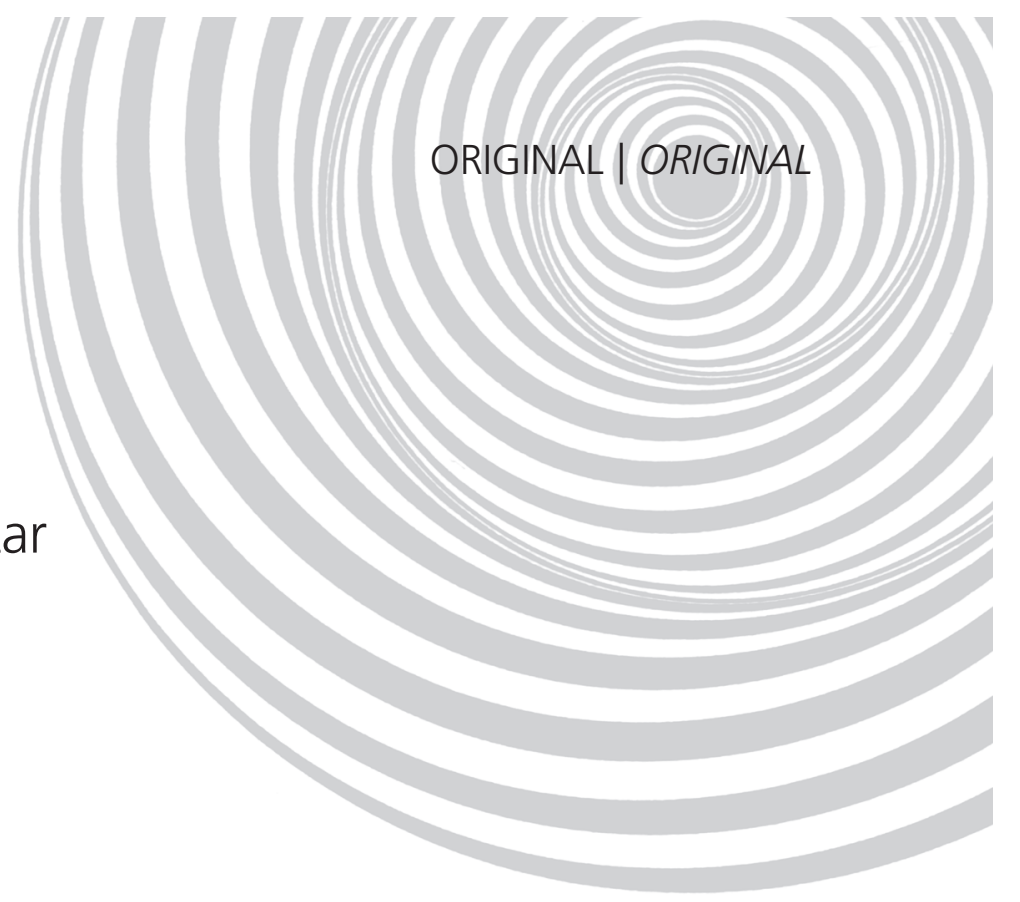

Ligia Mara Parreira SILVA ${ }^{1}$

Sônia Isoyama VENÂNCIO²

Dirce Maria Lobo MARCHIONI ${ }^{3}$

RE S U M O

\section{Objetivo}

Investigar a prevalência de consumo de alimentos complementares e os fatores associados à alimentação complementar oportuna em menores de um ano.

\section{Métodos}

Participaram do estudo 1176 crianças, durante a Campanha Nacional de Vacinação de 2003, em São Bernardo do Campo (SP), cujos acompanhantes responderam questionário que incluiu questões sobre a alimentação da criança nas 24 horas precedentes. A estimativa da prevalência de consumo dos alimentos complementares foi realizada por um modelo de regressão logística ajustado por idade; as medianas de introdução de alimentos por análise de sobrevida e os fatores associados à alimentação complementar oportuna por regressão de Poisson com ajuste robusto de variância e seleção hierarquizada de variáveis.

\section{Resultados}

Observou-se introdução precoce de alimentos complementares: no quarto mês, cerca de um terço das crianças recebiam suco de fruta e um quarto das crianças recebiam mingau, fruta ou sopa, ao passo que a probabilidade de consumir a comida da família aos oito meses foi baixa (48\%). A mediana de idade para o consumo de frutas foi de 266 dias (IC95\% 256-275), de papa de legumes foi 258 dias (IC95\% 250-264) e comida da família, 292 dias (IC 95\% 287-303). Os fatores associados ao consumo de alimentos sólidos antes dos seis meses de idade foram: sistema de assistência à saúde; idade materna; trabalho materno e uso de chupeta.

1 Faculdades Integradas de Santo André, Curso de Nutrição. Santo André, SP, Brasil.

2 Secretaria de Estado da Saúde de São Paulo, Instituto de Saúde. São Paulo, SP, Brasil.

${ }^{3}$ Universidade de São Paulo, Faculdade de Saúde Pública. R. Dr. Arnaldo, 715, 01246-904, São Paulo, SP, Brasil. Correspondência para/Correspondence to: D.M.L. MARCHIONI. E-mail: <marchioni@usp.br>. 
984 | L.M.P. SILVA et al.

\section{Conclusão}

O consumo precoce de alimentos sólidos, um risco potencial para a saúde infantil e para o desenvolvimento de doenças crônicas na idade adulta, evidenciam a necessidade de ações programáticas para reversão deste quadro.

Termos de indexação: Aleitamento materno. Alimentação complementar. Hábitos alimentares. Nutrição do lactente.

\section{A B S T R A C T}

\section{Objective}

This study investigated the complementary feeding practices and the factors associated with the appropriate timing of complementary feeding in children under one year of age.

\section{Methods}

The parents or caregivers of 1,176 children who attended the National Immunization Campaign in São Bernardo do Campo, Southeastern Brazil, in 2003 were interviewed to determine what the child was fed in the 24 hours prior to the interview. The prevalence of complementary food intake was estimated by a logistic regression model adjusted for age; the medians of the introduction of foods by survival analysis; and the factors associated with timely complementary feeding by Poisson regression with robust adjustment of variance and hierarchical variable selection.

\section{Results}

Complementary foods were introduced early: in the fourth month of age, nearly one-third of the children were already consuming fruit juices and one-fourth were consuming soft foods, fruits or soups. They were less likely to eat the same food as the family at eight months of age (48\%). The median age for fruit intake was 266 days (95\% Cl 256-275), for vegetable soup was 258 days (95\%Cl 250-264) and for family meals 292 days (95\% Cl 287-303). Factors associated with the intake of solid foods before age six months were: healthcare system, maternal age, maternal employment and use of pacifiers.

\section{Conclusion}

The early consumption of solid foods, a potential risk for the child's health and for the development of chronic diseases in adulthood, evidences the need for programmatic actions to reverse this situation.

Indexing terms: Breast feeding. Supplementary feeding. Food habits. Infant nutrition.

\section{N T R O D U ÇÃ O}

A alimentação adequada até os dois anos de idade é fundamental para promover o crescimento e o desenvolvimento apropriados da criança ${ }^{1}$. Até os seis meses de vida, o leite materno deve ser a única fonte alimentar, pois sozinho é capaz de nutrir adequadamente as crianças, além de favorecer a proteção contra doenças. Porém, a partir desse período, a complementação do leite materno é necessária para elevar a densidade energética da dieta e aumentar o aporte de micronutrientes ${ }^{2}$. Todos os alimentos, sólidos ou semi-sólidos oferecidos às crianças, à exceção do leite materno, são definidos como alimentos complementares $^{1,2}$.
A introdução dos alimentos na dieta da criança é uma etapa crítica que pode conduzir ao déficit nutricional e a enfermidades ${ }^{3}$. É um processo que envolve complexos fatores biológicos, culturais, sociais e econômicos que interferem no estado nutricional da criança ${ }^{1,2}$.

Em países em desenvolvimento, a dieta complementar tem um efeito imediato na saúde infantil, devido a fatores como a falta de acesso a alternativas adequadas ao leite materno; contaminação microbiana de alimentos ou líquidos e substituição do leite materno por alimentos menos nutritivos ${ }^{4}$. A introdução precoce dos alimentos complementares com frequência aumenta a vulnerabilidade da criança a diarreias, infecções 
e desnutrição ${ }^{5}$. A consequência imediata da má-nutrição durante esse período é o aumento da morbimortalidade e atraso no desenvolvimento mental e motor $1,2,4,5$.

Introduzir os alimentos complementares tardiamente também é desfavorável, pois pode ocorrer um retardo no crescimento da criança, além de aumentar o risco das deficiências nutricionais $^{3}$. Em casos individuais, a iniciação de alimentação complementar antes de seis meses pode ser recomendada, em especial quando as crianças não apresentam o crescimento satisfatório com amamentação exclusiva ${ }^{5}$. O Ministério da Saúde, em concordância com a Organização Mundial da Saúde, considera como alimentação complementar oportuna a introdução de alimentos sólidos ou semi-sólidos, de densidade energética mínima de $70 \mathrm{kcal} / 100 \mathrm{~mL}$ entre os seis e sete meses ${ }^{2,3,5}$.

Este trabalho tem como objetivo investigar as práticas de alimentação complementar e os fatores associados à introdução precoce de alimentos complementares no município de São Bernardo do Campo, em menores de um ano.

\section{MÉ T O D O S}

Esse estudo foi realizado utilizando-se dados de São Bernardo do Campo, do Projeto Amamentação e Municípios (AMAMUNIC), avaliação de práticas alimentares no primeiro ano de vida em dias nacionais de vacinação, desenvolvido pelo Instituto de Saúde da Secretaria de Estado da Saúde de São Paulo.

A população do estudo consistiu em 1176 crianças menores de um ano que compareceram aos 21 postos urbanos de vacinação do município, na $2^{\mathrm{a}}$ etapa da Campanha Nacional de Vacinação, em 2003.

O estudo foi aprovado pelo Comitê de Ética em Pesquisa da Faculdade de Saúde Pública da Universidade de São Paulo, protocolo $n^{\circ} 548$ em 11/09/01 e todos os participantes assinaram um Termo de Consentimento Livre e Esclarecido antes de sua inclusão na amostra.

\section{Amostragem}

Utilizou-se amostragem por conglomerados, com tamanho fixo de mil crianças. Considerando que as crianças não estavam distribuídas uniformemente nos postos de vacinação (conglomerados), adotou-se o sorteio em dois estágios, com probabilidade proporcional ao tamanho dos conglomerados. No primeiro estágio, foram sorteados os postos de vacinação, e, no segundo, foram sorteadas as crianças em cada posto, de forma sistemática. A amostra desenvolvida é considerada equiprobabilística ou autoponderada, pois se manteve constante a expressão $f=f_{1} \cdot f_{2^{\prime}}$ em que $f$ é a fração amostrada, $f_{1}$, o componente do primeiro estágio, e $\mathrm{f}_{2}$, o componente do segundo estágio. Os postos maiores tiveram maior probabilidade de ser sorteados no primeiro estágio $\left(f_{1}\right)$, e as crianças dos postos menores tiveram maior probabilidade de sorteio no segundo estágio $\left(f_{2}\right)$.

\section{Coleta e registro de dados}

A coleta de dados foi realizada por estudantes do curso de Nutrição de universidade privada da região; voluntários, previamente treinados pelas coordenadoras do projeto na semana anterior à coleta. Na ocasião do treinamento, receberam informações quanto aos objetivos da pesquisa; foram capacitados para a aplicação do instrumento para coleta dos dados; receberam orientações sobre o local horário e local de apresentação no dia do inquérito. Além disso, cada aluno recebeu um manual de apoio, com todas as orientações sobre a pesquisa e o contato com o supervisor de campo, para esclarecimento de eventuais dúvidas antes ou durante a coleta de dados. Foram alocados 64 entrevistadores nos 22 postos de vacinação sorteados.

O instrumento de coleta de dados utilizado foi elaborado pelo Instituto de Saúde de São Paulo, a partir das recomendações da Organização Mundial da Saúde ${ }^{6}$. O questionário foi composto por perguntas fechadas em sua maioria, e incluiu questões sobre a alimentação da criança nas 24 
horas precedentes. Pretende-se que a utilização dos dados atuais (current status) sobre alimentação infantil evite erros decorrentes da memória do informante. Também foram coletados dados sobre características maternas e das crianças.

A supervisão, realizada por médicas do sistema municipal de saúde, incluiu a ida a todos os postos de vacinação e a manutenção de plantão telefônico para o esclarecimento de dúvidas.

\section{Variáveis do estudo}

Foram investigadas:

Variáveis maternas: idade materna $(<20$ anos; 20 a 35 anos; $>35$ anos); escolaridade ( $<4$ anos; 4 a 8 anos; 9 a 11 anos; $\geq 12$ anos); paridade (variável dicotômica - primípara, sim ou não); tipo de parto (variável dicotômica - cesárea/vaginal); ter trabalhado na gravidez (variável dicotômica - sim/não); ter gozado licença maternidade (variável dicotômica - sim/não) e estar trabalhado atualmente (variável dicotômica - sim/não).

Variáveis da criança: idade (contínua, em dias); sexo (masculino/feminino); peso ao nascer (adequado, $\geq 2500 \mathrm{~g} /$ baixo peso, <2 $500 \mathrm{~g}$ ); uso de chupeta (variável dicotômica - sim/não); episódio de internação anterior (variável dicotômica - sim/não).

Variáveis socioeconômico/culturais: Região de São Bernardo do Campo onde foi vacinada (divisão administrativa do município, correspondente a cinco regiões geográficas), nascimento em Hospital Amigo da Criança (sim/não), tipo de serviço de assistência à saúde utilizado para puericultura (público/privado) e local de nascimento (no município; na região metropolitana de São Paulo, no Estado de São Paulo, fora do estado de São Paulo).

Para avaliação da prevalência de amamentação foram adotadas as seguintes categorias 6 :

- Aleitamento materno exclusivo: crianças alimentadas com leite materno e que não recebiam água, chá, suco ou outros alimentos, nas últimas 24 horas;
- Aleitamento materno predominante: crianças alimentadas com leite materno e água, chá ou suco, nas últimas 24 horas.

Variáveis da alimentação complementar: foi verificado o consumo de suco de frutas, frutas (na forma de papa ou suco de frutas); sopa ou papa de legumes; "comida de panela" (arroz e feijão, com ou sem carne).

Para avaliação da prática alimentar foram adotadas as seguintes categorias ${ }^{6}$.

- Alimentação complementar: introdução de alimentos sólidos ou semissólidos na alimentação da criança;

- Alimentação complementar oportuna: introdução de alimentos sólidos ou semissólidos entre seis e oito meses, na alimentação da criança.

\section{Análise estatística}

A estimativa da prevalência de consumo dos alimentos complementares foi realizada por um modelo de regressão logística, ajustado pela idade da criança.

Os fatores associados ao consumo de alimentos sólidos em menores de seis meses foram verificados por regressão de Poisson com ajuste robusto de variância e seleção hierarquizada de variáveis (Quadro 1). Nesta análise, somente participaram as crianças menores de seis meses $(n=625)$. Adotou-se como critério $p<5 \%$ para permanência no modelo final. Os resultados foram expressos como razão de prevalência.

O conjunto inicial foi composto pelas variáveis que, individualmente, apresentaram um $p$ descritivo do teste de $\chi^{2}$ de Person $<0,20$. Na análise múltipla, variáveis que foram significativas na etapa de entrada ( $p$ descritivo $<0,05$ ) permaneceram no modelo até o final, mesmo que tenham perdido sua significância em etapas posteriores.

Para a formação do modelo, estruturam-se inicialmente as variáveis mais frequentemente descritas na literatura como associadas à alimen- 
tação complementar, discriminando-as em blocos com seleção hierárquica, considerando três dimensões: dimensão dos processos estruturais da sociedade; dimensão dos processos do ambiente imediato da criança; e dimensão dos processos individuais da criança (Quadro 1).

Para a estimativa da mediana de introdução de frutas (em forma de suco ou papas), sopas e papas de legumes e comida da família utilizou-se a análise de sobrevida.

Foi utilizado o pacote estatístico STATA v.9.0.

\section{RES U LTADOS}

A amostra consistiu de 1176 crianças (50,6\% do sexo masculino). A maioria das crianças nasceu em hospitais públicos de São Bernardo do Campo, mas somente $30,9 \%$ dos partos ocorreram em Hospitais Amigos da Criança. O baixo peso ao nascer foi observado em 10,4\% das crianças e para grande parte delas não foram relatados episódios de internação após o nascimento. Foi grande a porcentagem de crianças que utilizavam chupeta. Considerando as características mater- nas, a maioria possuía até 35 anos, tinha até 11 anos de estudo e não estava trabalhando no momento da entrevista. Foi homogênea a distribuição de mães primíparas e multíparas e a maioria dos partos foi do tipo cesárea (Tabela 1).

A estimativa da prevalência de aleitamento materno exclusivo aos 120 dias foi de $15 \%$ e aos 180 dias foi de $5 \%$. Na modalidade predominante aos 120 e 180 dias, foram estimadas prevalências de $34 \%$ e $13 \%$, respectivamente (Figura 1).

A mediana de idade para o consumo de frutas (suco/papa) foi de 266 dias (IC95\% 256-275) dias, de papa de legumes foi 258 dias (IC95\% 250-264) e comida da família 292 dias (IC 95\% 287-303).

A Tabela 2 apresenta a prevalência estimada para o consumo de alimentos complementares aos quatro e aos oito meses. Pode-se observar que os alimentos complementares foram precocemente introduzidos na dieta dos lactentes: cerca de um terço das crianças recebiam suco de fruta e $1 / 4$ das crianças recebiam mingau, fruta ou sopa aos quatro meses de vida, ao passo que a probabilidade de consumir "comida de panela" aos oito meses foi baixa (48\%). Por "comida de panela" entende-se, nesta população de estudo,

Quadro 1. Modelo conceitual com seleção hierárquica para determinação da prevalência alimentação complementar em crianças menores de um ano. São Bernardo do Campo (SP), 2003.

1. Dimensão dos processos estruturais da sociedade

2. Dimensão dos processos do ambiente imediato da criança

3. Dimensão dos processos individuais da criança

Bloco individual

Peso nascimento

Episódio de

Internação anterior

Uso de chupeta

Sexo

\section{Geopolítico}

Região SBC onde vacinou

Sócio-cultural

Escolaridade materna

Idade materna

Paridade

Tipo de parto

Trabalho na gravidez

Licença maternidade

Trabalho atual
Acesso saúde

Hospital amigo da criança

Tipo serviço saúde

Local de nascimento

AC: alimentação complementar; SBC: São Bernardo do Campo. 
988 | L.M.P. SILVA et al.

Tabela 1. Distribuição do número e porcentagem de crianças menores de um ano, segundo variáveis de estudo. São Bernardo do Campo (SP), 2003.

\begin{tabular}{|c|c|c|}
\hline Variável & $\mathrm{N}$ & $\%$ \\
\hline \multicolumn{3}{|l|}{ Sexo } \\
\hline Masculino & 595 & 50,6 \\
\hline Feminino & 581 & 49,4 \\
\hline \multicolumn{3}{|l|}{ Idade (dias) } \\
\hline $0 \quad \mid-120$ & 394 & 33,5 \\
\hline $120-180$ & 244 & 20,8 \\
\hline $180-\mid 300$ & 380 & 32,3 \\
\hline $300-1365$ & 158 & 13,4 \\
\hline \multicolumn{3}{|l|}{ Peso ao nascer ${ }^{*}$} \\
\hline Baixo peso $(<2500 \mathrm{~g})$ & 117 & 10,4 \\
\hline Peso adequado ( $\geq 2500 \mathrm{~g})$ & 1012 & 89,6 \\
\hline \multicolumn{3}{|l|}{ Hospital amigo da criança* } \\
\hline Não & 770 & 69,1 \\
\hline Sim & 345 & 30,9 \\
\hline \multicolumn{3}{|c|}{ Tipo de serviço de assistência à saúde* } \\
\hline Privado & 498 & 42,7 \\
\hline Público & 668 & 57,3 \\
\hline \multicolumn{3}{|l|}{ Internação* } \\
\hline Não & 1009 & 87,2 \\
\hline Sim & 148 & 12,8 \\
\hline \multicolumn{3}{|c|}{ Local de nascimento da criança* } \\
\hline São Bernardo do Campo & 706 & 60,7 \\
\hline Outros & 457 & 39,3 \\
\hline \multicolumn{3}{|l|}{ Uso de chupeta* } \\
\hline Não & 600 & 51,6 \\
\hline Sim & 564 & 48,4 \\
\hline \multicolumn{3}{|l|}{ Idade materna (anos) ${ }^{*}$} \\
\hline$<20$ & 136 & 13,6 \\
\hline $201-35$ & 749 & 75,0 \\
\hline$\geq 35$ & 114 & 11,4 \\
\hline Total & 999 & 100,0 \\
\hline \multicolumn{3}{|c|}{ Escolaridade materna (anos)* } \\
\hline$\leq 4$ & 94 & 9,5 \\
\hline 5 a 8 & 322 & 32,6 \\
\hline 9 a 11 & 454 & 46,0 \\
\hline$\geq 12$ & 117 & 11,9 \\
\hline \multicolumn{3}{|l|}{ Tipo de parto* } \\
\hline Normal & 446 & 38,4 \\
\hline Outro (fórceps, cesárea) & 714 & 61,6 \\
\hline \multicolumn{3}{|l|}{ Primipara* } \\
\hline Sim & 495 & 49,6 \\
\hline Não & 503 & 50,4 \\
\hline \multicolumn{3}{|l|}{ Trabalho atual ${ }^{*}$} \\
\hline Não & 774 & 77,6 \\
\hline Sim & 223 & 22,4 \\
\hline
\end{tabular}

*Variáveis com dados faltantes.
Tabela 2. Probabilidade de consumo de alimentos complementares estimada por modelo de regressão logística ajustado pela idade da criança (dias). São Bernardo do Campo (SP), 2003.

\begin{tabular}{lcc}
\hline \multirow{2}{*}{ Alimentos } & \multicolumn{2}{c}{ Probabilidade de consumo (\%) } \\
\cline { 2 - 3 } & Aos 4 meses & Aos 8 meses \\
\hline Suco de fruta & 29 & 77 \\
Fruta & 25 & 64 \\
Mingau & 25 & 49 \\
Sopa ou papa de legume & 25 & 78 \\
"Comida de panela" & 9 & 48 \\
\hline
\end{tabular}

as preparações básicas e habituais (tipo "staple food") preparada para a família, significando, especialmente, arroz e feijão.

Na análise multivariada, a influência dos fatores associados ao consumo de alimentos sólidos em menores de seis meses foi investigada de acordo com a sua relação hierárquica aos outros fatores no modelo como mostrado na Tabela 3.

Observou-se menor risco para consumo de alimentos sólidos antes do período recomendado em crianças atendidas nos serviços públicos de assistência à saúde e que não usavam chupetas, e maior risco em crianças cujas mães relataram estar trabalhando no momento da entrevista e com idade superior a 35 anos.

\section{I S C U S S Ã O}

Este estudo investigou as práticas alimentares das crianças no primeiro ano de vida de São Bernardo do Campo, município da região metropolitana de São Paulo, e fatores associados ao consumo de alimentos sólidos nos primeiros seis meses de idade. Verificou-se interrupção prematura da amamentação exclusiva e introdução precoce de alimentos complementares, em desacordo com as pautas recomendadas por órgãos nacionais e internacionais de saúde ${ }^{1,5}$.

Nosso estudo corrobora com outros realizados no Brasil e Argentina que identificaram a introdução precoce de alimentos complementares em menores de um ano ${ }^{7-16}$. A introdução precoce de alimentos também é ocorrência frequente em 


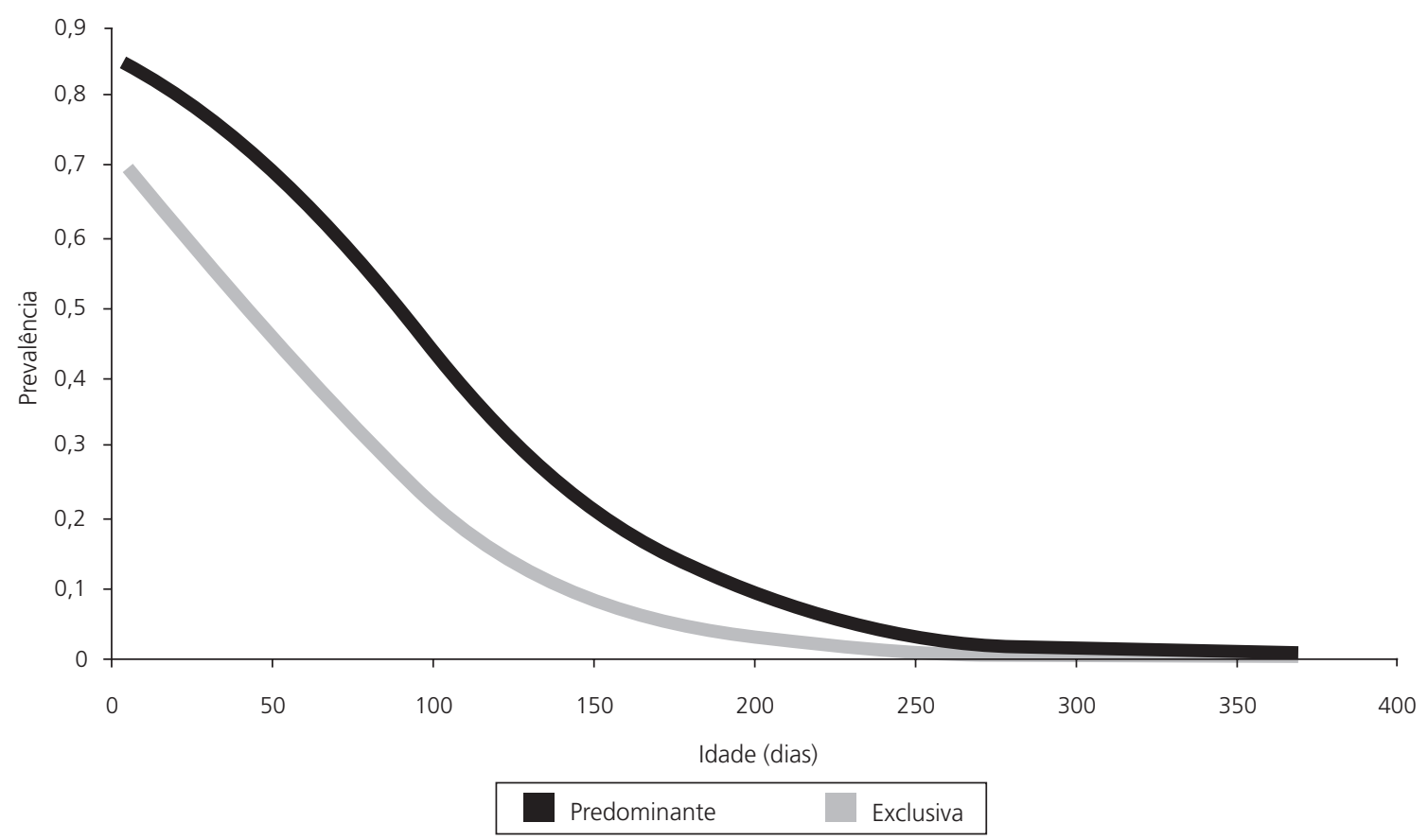

Figura 1. Prevalências estimadas de amamentação exclusiva e predominante de crianças menores de um ano. São Bernardo do Campo (SP), 2003

Tabela 3. Razão de prevalência e respectivos intervalos de confiança (IC 95\%) para variáveis associadas ao consumo de alimentos sólidos em crianças menores de seis meses, conforme modelo de regressão de Poisson com seleção hierárquica de variáveis. São Bernardo do Campo (SP), 2003.

\begin{tabular}{|c|c|c|}
\hline Variáveis & Razão de prevalência ${ }^{1}$ & Intervalos de confiança 95\% \\
\hline & Bloco 1 - Dimensão dos processos est & \\
\hline \multicolumn{3}{|c|}{ Tipo de assistência a saúde } \\
\hline Pública & 1 & \\
\hline \multirow[t]{2}{*}{ Privado } & 1,25 & $1,01-1,54$ \\
\hline & Bloco 2 - Dimensão dos processos do & ança \\
\hline \multicolumn{3}{|l|}{ Idade materna ${ }^{2}$} \\
\hline$<20$ anos & 1 & \\
\hline $20 \mid-35$ anos & 1,30 & $0,87-2,07$ \\
\hline$\geq 35$ anos & 2,57 & $1,00-2,78$ \\
\hline \multicolumn{3}{|l|}{ Trabalho atua/2 } \\
\hline Não & 1 & \\
\hline \multirow[t]{2}{*}{ Sim } & 1,28 & $1,00-1,65$ \\
\hline & Bloco 3 - dimensão dos processos ind & \\
\hline \multicolumn{3}{|c|}{ Uso de chupeta ${ }^{3}$} \\
\hline Sim & 1 & \\
\hline Não & 0,63 & $0,50-0,81$ \\
\hline
\end{tabular}

${ }^{1}$ Modelo multivariado de regressão de Poisson.

${ }^{2}$ Ajustado por assistência à saúde.

${ }^{3}$ Ajustado por assistência á saúde, idade materna e trabalho atual. 
países de diferentes partes do mundo e foi relatada em estudos com diversos delineamentos, como na Europa, em estudo transversal realizado em cinco países (Alemanha, Bélgica, Itália, Polônia e Espanha), entre 2002 e 2004 17; no Perth Infant Feeding Study, estudo de coorte conduzido na Austrália ( $n=5$ 192), onde $93 \%$ das crianças tinha recebido seu primeiro alimento sólido antes de 36 semanas de idade ${ }^{18}$ e no Infant Feeding Practices Study II, conduzido nos Estados Unidos ${ }^{19}$. O consumo precoce de alimentos complementares traz consequências danosas para a saúde da criança, como aumento de risco para as doenças infecciosas, particularmente as diarreias, em consequência de uma menor ingestão dos fatores de proteção existentes no leite materno ${ }^{2,4}$. Além disso, as evidências têm se acumulado mostrando a relação entre consumo de alimentos complementares antes dos seis meses de idade e aumento de risco para obesidade e doenças cardiovasculares na idade adulta ${ }^{20}$.

A proporção de crianças recebendo frutas aos quatro meses de idade, de $25 \%$ no presente estudo, foi superior ao observado nos Estados Unidos $^{19}$, de $17 \%$, no Infant Feeding Practices Study II, e ao observado na Bavária, Alemanha, de $16,4 \%{ }^{21}$. Apesar de inadequada, a introdução precoce de frutas, parece ocorrer pela ênfase dada pelos profissionais de saúde, facilidade de aceitação da criança e oferta do produto ${ }^{8,22}$.

A criança, após os seis meses de idade, deve receber de forma gradual outros alimentos, sendo necessário garantir o aporte energético com uma alimentação de elevada densidade energética e rica em micronutrientes ${ }^{1,4,5}$. Recomenda-se iniciar gradativamente os alimentos e concomitantemente modificar sua consistência até chegar à alimentação da família ${ }^{5}$. Notou-se que, entre as crianças maiores de sete meses, a probabilidade de receberem a alimentação da família foi de $34 \%$, porcentagem inferior a encontrada por Saldiva et al. ${ }^{22}$, que foi de $39 \%$ e de Audi et al. ${ }^{13}$, que foi de $41 \%$, e também inferior ao reportado por Gatica et al. ${ }^{16}$, na Argentina, de $64 \%$ aos seis meses de idade.

Neste estudo, não foram identificados fortes fatores preditores para o consumo precoce de alimentos sólidos, similar a estudos com o mesmo objetivo conduzidos no Brasil|23,24. Verificou-se maior risco no grupo de crianças que utilizavam o sistema privado de assistência à saúde. No Brasil a assistência pública e privada de atenção à saúde coexistem, configurando, diferenciadamente, questões referentes a acesso, fato que pode ser considerado um legado de políticas prévias à implantação do Sistema Único de Saúde (SUS). Esta duplicidade se expressa no próprio texto constitucional que estabeleceu o SUS. No entanto, ao longo das duas últimas décadas, tem ocorrido a ampliação da rede pública, principalmente a ambulatorial, que aponta tanto o esforço para universalização da assistência a partir da garantia de acesso à atenção primária e de reversão do modelo assistencial centrado no atendimento hospitalar ${ }^{25}$, o que pode explicar o achado deste estudo.

Também, maior risco foi observado em crianças cujas mães estavam trabalhando na época da entrevista, similar a estudo conduzido em Florianópolis ${ }^{26}$. A industrialização e a inserção da mulher no mercado de trabalho resultaram em alteração nos hábitos alimentares da família, contexto que provavelmente teve impacto na alimentação infantili ${ }^{8}$, como o observado no presente artigo.

A idade foi outro fator preditor para introdução precoce de alimentos. Filhos de mães mais velhas (acima de trinta anos) tiveram risco 2,5 vezes maior que mães mais jovens (menos de vinte anos) para consumo de alimentos complementares precocemente, resultados convergentes com um levantamento por telefone, de base populacional no Canadá, mas contrários ao reportado nos estudos transversais de base populacional conduzidos na Bavária, Alemanha ${ }^{21}$, na Suiça ${ }^{27}$, e em estudos de seguimento na Europa ${ }^{17}$ e na Austrália ${ }^{18}$.

O hábito de não usar chupeta associou-se inversamente com a introdução precoce da alimentação sólida, consistente com estudos realizados no Brasil que demonstram que o uso de chupeta associa-se negativamente ao tempo de aleitamento materno exclusivo ${ }^{13,28-30}$. 
Apesar da recomendação de que o leite materno deve ser o único alimento a ser oferecido às crianças menores de seis meses, baixo percentual delas ainda se beneficia dessa prática. No presente artigo a estimativa da prevalência de amamentação exclusiva foi de apenas $5 \%$, semeIhante à encontrada por Chaves et al. ${ }^{28}$ e menor a encontrada por Carvalhaes et al. ${ }^{29}$ e França et al..$^{30}$. As evidências para efeito protetor do aleitamento para doenças cardiovasculares e seus fatores de risco se acumulam ${ }^{31}$.

Por se tratar de estudo transversal, o estudo apresenta como limitação a impossibilidade de identificar a idade de início do consumo de cada alimento. $\mathrm{O}$ instrumento utilizado para avaliar a alimentação nas $24 \mathrm{~h}$ precedentes à entrevista não possibilitam identificar as quantidades e a frequência de consumo dos alimentos. A cuidadosa seleção da amostra em Campanha de Vacinação congregou alto percentual de crianças e mostrou-se efetiva em garantir a representatividade do município. Deve-se ressaltar ainda a lacuna representada pela ausência de informação sobre o consumo de alimentos industrializados, apesar do reconhecimento que o consumo destes alimentos representa um erro alimentar. No delineamento do estudo, optou-se por limitar a investigação aos alimentos básicos e in natura, em decorrência do constrangimento de tempo e características do espaço para coleta de dados, qual seja, a fila de espera para vacinação.

\section{ONCLUSÃO}

Os resultados deste estudo permitiram conhecer as características alimentares dos menores de um ano e fatores associados à alimentação complementar. Observou-se uma baixa prevalência de amamentação exclusiva e consumo precoce de alimentos sólidos em elevada proporção nas crianças estudadas, com risco potencial para sua saúde e para o desenvolvimento de doenças crônicas na idade adulta. Os fatores associados ao consumo de alimentos sólidos antes dos seis meses de idade foram: sistema de assistência à saúde; idade materna; trabalho materno e uso de chupeta.
Estudos para avaliar as práticas de alimentação infantil devem ser realizados, pois são importante fonte de informação para o planejamento, avaliação e implementação de políticas e programas dirigidos a apoiar e promover o aleitamento materno e a introdução dos alimentos complementares em idade oportuna.

\section{COLABORADORES}

L.M.P. SILVA colaborou com a análise dos dados e redação do manuscrito; SI. VENÂNCIO colaborou com o delineamento do estudo e orientação da redação do manuscrito. D.M.L. MARCHIONI coloborou com a supervisão do trabalho de campo, análise dos dados, orientação e participação na redação do manuscrito.

\section{REFERÊ NCIAS}

1. Agostoni $C$, Decsi T, FewtrellM, Goulet O, Kolacek S, Koletzko B, et al. Complementary feeding: a commentary by the ESPGHAN Committee on Nutrition. J Pediatr Gastroenterol Nutr. 2008; 46(1): 99-110.

2. World Health Organization. The optimal duration of exclusive breastfeeding: a systematic review. Geneva: World Health Organization; 2001.

3. World Health Organization. Multicentre Growth Reference Study Group. Complementary feeding in the WHO Growth Reference Study. Acta Paediatr. 2006; 95(450 Suppl):27-37.

4. Giugliani ERJ, Victora, CG. Complementary feeding. J Pediatr (Rio J). 2000; 76(Supl. 3):253-62.

5. Brasil. Ministério da Saúde. Secretaria de Política de Saúde. Guia Alimentar para crianças menores de 2 anos normas e manuais técnicos, n. 107. Brasília: Ministério da Saúde; 2002.

6. World Health Organization. Informal meeting to review and develop indicators for complementary feeding. Report of an Informal Meeting 3-5; December 2002; Washington, DC [cited 2010 May 23 ]. Available from: <http://whqlibdoc.who. int/hq/2002/a91059.pdf>.

7. Marchioni DML, Latorre MRD, Szarfarc SC, Souza SB. Complementary feeding: study on prevalence of food intake in two health centers of São Paulo City. Arch Latinoam Nutr. 2001; 51(2):161-6.

8. Parada CMGL, Carvalhaes MABL, Jamas MT. Práticas de alimentação complementar em crianças no primeiro ano de vida. Rev Latino-am Enfermagem. [Internet] 2007; [acesso 2010 maio 13]; 15(2). Disponível em: <http://www.scielo.br/pdf/rlae/v15 n2/pt_v15n2a14.pdf>. 
9. Brunken GS, Silva SM, França GVA, Escuder MM, Venâncio SI. Fatores associados à interrupção precoce do aleitamento materno exclusivo e à introdução tardia da alimentação complementar no centro-oeste brasileiro. J Pediatr (Rio J). 2006; 82(6): 445-51.

10. Figueiredo MG, Sartorelli DS, Zan TAB, Garcia E, Silva LC, Carvalho FLP, et al. Inquéritos de avaliação rápida das práticas de alimentação infantil em São José do Rio Preto, São Paulo, Brasil. Cad Saúde Pública. 2004; 20(1):172-9.

11. Silveira FJF, Lamounier JA. Prevalência do aleitamento materno e práticas de alimentação complementar em crianças com até 24 meses de idade na região do Alto Jequitinhonha, Minas Gerais. Rev Nutr. 2004; 17(4): 437-47. doi: 10.1590/S1415-52 732004000400004 .

12. Simon VGN, Souza JMP, Souza SB. Introdução de alimentos complementares e sua relação com variáveis demográficas e socioeconômicas, em crianças no primeiro ano de vida, nascidas em Hospital Universitário no município de São Paulo. Rev Bras Epidemiol. 2003; 6(1):29-38.

13. Audi, CAF, Corrêa AMS, Latorre MRDO. Alimentos complementares e fatores associados ao aleitamento materno e ao aleitamento materno exclusive em lactentes até 12 meses de vida em Itapira, São Paulo, 1999. Rev Bras Saúde Mater Infant. 2003; 3(1):85-93.

14. Alberico APM, Veiga GV, Baião MR, Santos MMAS, Souza SB, Szarfarc SC. Breast-feeding, weaning diet and iron deficiency anaemia in infants. Nutr Food Sci. 2003; 33(3):111-19.

15. Caetano MC, Ortiz TT, da Silva SG, de Souza FI, Sarni RO. Complementary feeding: inappropriate practices in infants. J Pediatr (Rio J) [Internet]. 2010 [acesso 2010 maio 13]; 86(3). Disponível em: <http://www.scielo.br. doi: 10.1590/S0021-75572 01000300006.

16. Gatica Cl, Méndez de Feu MC; Investigadores participantes de Filiales de La 4ta Región Centro Cuyo de la Sociedad Argentina de Pediatría. Feeding practices in children aged under 2 years. Arch Argent Pediatr. 2009; 107(6):496-503.

17. Schiess S, Grote V, Scaglioni S, Luque V, Martin F, Stolarczyk A, et al. European Childhood Obesity Project. Introduction of complementary feeding in 5 European countries. J Pediatr Gastroenterol Nutr. 2010; 50(1):92-8.

18. Scott JA, Binns CW, Graham KI, Oddy WH. Predictors of the early introduction of solid foods in infants: results of a cohort study. BMC Pediatr. [Internet]. 2009 [cited 2010 May 13]; 9:60. Available from: <http://www.biomedcentral.com>/ 1471-2431/9/60>. doi:10.1186/1471-2431-9-60.

19. Fein SB, Labiner-Wolfe J, Scanlon KS, GrummerStrawn LM. Selected complementary feeding practices and their association with maternal education. Pediatrics. 2008;122 (Suppl 2):S91-7.

20. Wu TC, Chen PH. Health consequences of nutrition in childhood and early infancy. Pediatr Neonatol. 2009; 50(4):135-42.

21. Rebhan B, Kohlhuber M, Schwegler U, Koletzko $\mathrm{BV}$, Fromme $\mathrm{H}$. Infant feeding practices and associated factors through the first 9 months of life in Bavaria, Germany. J Pediatr Gastroenterol Nutr. 2009; 49(4):467-73.

22. Saldiva SRDM, Escuder MM, Mondini L, Levy RB, Venancio SI. Práticas alimentares de crianças de 6 a 12 meses e fatores maternos associados. J Pediatr (Rio J). 2007; 83(1):53-58.

23. Santos-Neto $E T$, Faria $C P$, Barbosa $M L$, Oliveira $A E$, Zandonade E. Association between food consumption in the first months of life and socioeconomic status: a longitudinal study. Rev Nutr. 2009; 22(5):675-85. doi: 10.1590/S1415-52 732009000500008.

24. Simon VGN, Souza JMP, Souza SB. Introdução de alimentos complementares e sua relação com variáveis demográficas e socioeconômicas, em crianças no primeiro ano de vida, nascidas em Hospital Universitário no município de São Paulo. Rev Bras Epidemiol. 2003; 6(1):29-38.

25. Menicucci TMG. O Sistema Único de Saúde, 20 anos: balanço e perspectivas. Cad Saúde Pública. 2009; 25(7):1620-5.

26. Corrêa EN, Corso ACT, Moreira EAM, Kazapi IAM. Alimentação complementar e características maternas de crianças menores de dois anos de idade em Florianópolis (SC). Rev Paul Pediatr. 2009; 27(3):258-64.

27. Dratva J, Merten S, Ackermann-Liebrich U. The timing of complementary feeding of infants in Switzerland: compliance with the Swiss and the WHO guidelines. Acta Paediatr. 2006; 95(7): 818-25.

28. Chaves RG, Lamounier JA, César CC. Fatores associados com duração da amamentação. J Pediatr (Rio J). 2007; 83(3):241-6.

29. Carvalhaes MABL, Parada CML, Costa MP. Factors associated with exclusive breastfeeding in children under four months old in Botucatu-SP, Brazil. Rev Latino-am Enfermagem. 2007; 15(1):62-9.

30. França GVA, Brunken GS, Silva SM, Escuder MM, Venancio SI. Determinantes da amamentação no primeiro ano de vida em Cuiabá, Mato Grosso. Rev Saúde Pública. 2007; 41(5):711-8.

31. Singhal A. Early nutrition and long-term cardiovascular health. Nutr Rev. 2006; 64(5 Pt 2): S44-9.

Recebido em: 11/1/2009

Versão final reapresentada em: 26/5/2010 Aprovado em: 7/6/2010 